\title{
ERRATUM
}

\section{Mitochondrial DNA analysis of Yayoi period human skeletal remains from the Doigahama site}

Kazunari Igawa, Yoshitaka Manabe, Joichi Oyamada, Yoshikazu Kitagawa, Katsutomo Kato, Kazuya Ikematsu, Ichiro Nakasono, Takayuki Matsushita and Atsushi Rokutanda

Journal of Human Genetics (2009) 54, 753; doi:10.1038/jhg.2009.126

Correction to: Journal of Human Genetics (2009) 54, 581-588; doi:10.1038/jhg.2009.81; published online 21 August 2009

Since the publication of the above paper, the authors noticed an error within the Results and Discussion heading, under the subheading 'Phylogenetic analysis based on frequency distributions of DNA sequence type'.
The correct sentence is given below:

In all, 5, 2, 1, 22, 1 and 3 individuals from the 2500-year-old Linzi site belonged to groups I, II, III, IV, V and VI, respectively.

Our typesetters would like to apologize for this mistake. 